\title{
Mutagénesis dirigida del conector interdominio ácido FVIIla3 del factor VIII de la coagulación como estrategia para favorecer la cristalización de sus complejos con la trombina Site-directed mutagenesis of the acidic interdomain linker FVIIla3 of the coagulation factor VIII as a strategy to favor crystallization of its complexes with thrombin
}

Jeremy Rojas-Murcia'1, Silvia Arce-Solano², Pablo Fuentes-Prior ${ }^{3}$, Erick Hernández-Carvajal ${ }^{4}$

Rojas-Murcia, J; Arce-Solano, S; Fuentes-Prior, P; Hernández-Carvajal, E. Mutagénesis dirigida del conector interdominio ácido FVIIla3 del factor VIII de la coagulación como estrategia para favorecer la cristalización de sus complejos con la trombina. Tecnología en Marcha. Vol. 32-4. Octubre-

Diciembre 2019. Pág 39-50.

doi) https://doi.org/10.18845/tm.v32i4.4789

2 Investigadora Instructora. Ing. Licenciada en Biotecnología. Centro de Investigación en Biotecnología Instituto Tecnológico de Costa Rica. Costa Rica.

(iD) https://orcid.org/0000-0003-4357-3661

3 Investigador Titular. Doctor en Bioquímica. Institut d'Investigació Biomèdica (IIB) Sant Pau, Hospital de la Santa Creu i Sant Pau. Barcelona, España. (iD https://orcid.org/0000-0002-6618-3204

4 Investigador Asociado. Doctor en Biomedicina. Centro de Investigación en Biotecnología. Instituto Tecnológico de Costa Rica. Costa Rica. Correo electrónico: erhernandez@itcr.ac.cr. 


\title{
Palabras clave
}

Enfermedades cardiovasculares; trombina; factor VIII; mutagénesis dirigida; cristalización de proteínas.

\section{Resumen}

Las enfermedades cardiovasculares representan una de las principales causas de morbilidad y mortalidad a nivel mundial, lo que se traduce en un fuerte impacto económico en el sector salud. El factor VIII (FVIII) es un cofactor esencial en los procesos hemostáticos que participa en la formación del coágulo ante alguna señal de daño vascular. La proteasa trombina es el principal activador fisiológico del FVIII, pero los mecanismos moleculares de reconocimiento del FVIII por la trombina y la formación de los complejos transitorios Michaelis-Menten correspondientes aún no se han descrito en detalle. La cristalografía de rayos $X$ es una técnica que permite resolver la estructura 3D de complejos proteicos, pero requiere la cristalización previa de la muestra a estudiar. Con el objetivo de favorecer la formación de cristales del FVIIla3 humano (residuos Glu ${ }^{1649}$-Arg ${ }^{1689}$ ) acomplejado con la trombina, se realizó mutagénesis dirigida para generar los doble-mutantes FVIIla3 (R1689Q, G1690P) y FVIIla3 (R1689G, G1690P). Estos fragmentos fueron sobreexpresados de forma heteróloga y purificados, obteniendo rendimientos de $\sim 1.5$ mg por cada litro de cultivo bacteriano. Esto permitió generar los complejos proteicos FVIIla3 (R1689Q, G1690P)•trombina y FVIIla3 (R1689G, G1690P)•trombina en cantidades suficientes para explorar sus espacios de solubilidad de forma extensiva. En total, en cada caso se evaluaron entre 630 y 820 condiciones de cristalización distintas. A partir de esta búsqueda inicial se obtuvo algún tipo de precipitado cristalino en 22 condiciones, 10 de las cuales lograron ser optimizadas para obtener monocristales de alta calidad para posteriores ensayos de difracción con rayos $\mathrm{X}$.

\section{Keywords}

Cardiovascular diseases; thrombin; factor VIII; site-directed mutagenesis; protein crystallization.

\begin{abstract}
Cardiovascular diseases are one of the most important causes of morbidity and mortality worldwide, which translates into a strong economic impact in the health sector. Factor VIII (FVIII) is an essential cofactor within the hemostatic processes involved in blood clot formation upon vascular damage. The proteinase thrombin is the most important physiological activator of FVIII, but the molecular mechanisms underlying recognition of FVIII by thrombin and the formation of the corresponding, transient Michaelis-Menten complexes are still only poorly understood. X-ray crystallography allows the resolution of the 3D structure of protein complexes, but a prerequisite is the crystallization of the sample to be analyzed. To grow crystals of the human FVIIla3 (residues Glu ${ }^{1649}$-Arg ${ }^{1689}$ ) complexed with thrombin, site-directed mutagenesis was performed to generate the double-mutants FVIIla3 (R1689Q, G1690P) and FVIIla3 (R1689G, G1690P). Both fragments were overexpressed as heterologous proteins and purified with yields of $\sim 1.5 \mathrm{mg}$ per liter of bacterial culture. Availability of these recombinant proteins allowed in turn generation of FVIIla3 (R1689Q, G1690P)•thrombin and FVIIla3 (R1689G, G1690P)•thrombin complexes to perform extensive screenings of their solubility space. Altogether, a total of 630 to 820 different crystallization conditions were evaluated for both complexes. From these initial screen, some sort of crystalline precipitate was obtained in 22 conditions, 10 of which could be optimized to grow high-quality single crystals for subsequent $\mathrm{X}$-ray diffraction analysis.
\end{abstract}




\section{Introducción}

Las enfermedades cardiovasculares (ECV) representan una de las principales causas de morbilidad y mortalidad a nivel mundial. En España, aproximadamente uno de cada tres decesos es causado por una ECV, esto se traduce en un elevado coste al sistema sanitario de más de 9.000 millones de euros al año [1]. De forma similar, según un estudio de la Organización Mundial de la Salud (OMS) publicado en el 2014 [2], en Costa Rica el 30\% de las muertes son debido a ECVs, tanto en hombres como en mujeres de entre 30 y 70 años. Dentro de este grupo de enfermedades se encuentran las isquemias cardíacas o infartos y los derrames cerebrovasculares o ictus, los cuales en muchos de los casos se deben a eventos de trombosis, caracterizados por la formación de coágulos de sangre que se alojan en los vasos sanguíneos en el corazón o en el cerebro, interrumpiendo el suministro de oxígeno y nutrientes en estos órganos [1]. El proceso de formación del coágulo es complejo y altamente regulado por un gran número de proteínas que interaccionan entre sí y con células como las plaquetas. Un elemento esencial de este proceso de coagulación es la activación de la protrombina a trombina, la cual es considerada una de las proteasas con actividad procoagulante más importante, ya que participa en la activación del fibrinógeno, del factor XIII y de receptores activados por proteasas (PARs) en la superficie de las plaquetas, así como de los cofactores $\mathrm{V}$ y VIII, favoreciendo la formación del coágulo [3]. La activación de estas moléculas, mediada por la trombina, requiere de una serie de cortes proteolíticos específicos, gracias a la formación de complejos transitorios con los sustratos correspondientes [4]. En el caso del FVIII, estudios bioquímicos sugieren que las secuencias interdominio del FVIII (conocidas como conectores a1, a2 y a3) presentan características específicas que les permiten orientar los sitios de corte y activación del factor VIII hacia el sitio activo de la trombina, favoreciendo la proteólisis y por tanto la activación del cofactor. Sin embargo, actualmente no existe suficiente información a nivel atómico-molecular sobre los mecanismos moleculares detallados de interacción del FVIII con la trombina, lo que impide su utilización como diana para el desarrollo de fármacos antitrombóticos novedosos, capaces de tratar con éxito las ECVs. La cristalografía de proteínas mediante rayos X, es la técnica más utilizada para resolver las estructuras tridimensionales (3D) de macromoléculas a nivel atómico [5]. Resolver la estructura del complejo trombina-FVIII permitirá comprender mejor el mecanismo de activación por proteólisis del FVIII mediado por la trombina, pero para ello se requiere crecer monocristales del complejo proteico para la posterior colecta de datos de difracción de rayos $X$. Con el objetivo de fomentar la obtención de monocristales del conector FVIIIa3 acomplejado con la proteinasa humana, en este trabajo se realizó mutagénesis dirigida de residuos específicos del FVIIla3 para la sobreexpresión y purificación de los doble-mutantes FVIIla3 (R1689Q, G1690P) y FVIIla3 (R1689G, G1690P).

\section{Materiales y métodos}

Mutagénesis dirigida: A partir de la confirmación de la clonación en el vector de expresión pET$3 a$ de la secuencia del FVIIla3 (residuos aminoacídicos 1649-1689), fusionada a una cola de histidinas en el extremo N-terminal, se procedió a diseñar in silico la mutagénesis dirigida para la obtención de los doble-mutantes FVIIla3 (R1689Q, G1690P) y FVIIla3 (R1689G, G1690P). Las secuencias de los fragmentos respectivos, junto a algunas de sus características fisicoquímicas relevantes se presentan en el cuadro 1.

Empleando la secuencia nucleotídica teórica del FVIIla3, se realizó el diseño de los imprimadores requeridos para generar el FVIIla3 (R1689Q, G1690P) y FVIIla3 (R1689G,

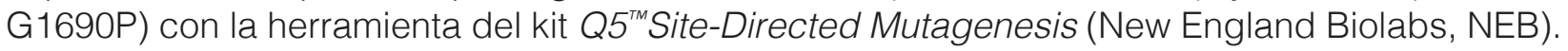
La síntesis de los imprimadores fue contratada a Macrogen (Korea). Para ambos fragmentos doble-mutantes del FVIIla3 se llevó a cabo un protocolo de mutagénesis dirigida, siguiendo las 
recomendaciones indicadas en el kit Q5 Site-Directed Mutagenesis (NEB). Posteriormente, se realizó una transformación de las células competentes E. coli NEB 5-alpha, y para comprobar la generación del mutante deseado, se realizó una extracción del ADN plasmídico empleando el kit de purificación QIAprep Spin Miniprep (Qiagen). Las muestras de ADN plasmídico extraídas por triplicado, se cuantificaron en un espectrofotómetro (NanoDrop ${ }^{\mathrm{TM}}$ Lite), se visualizaron en un gel de agarosa al 1\%, y se secuenciaron para confirmar la secuencia mutante de interés (Macrogen, Korea).

Cuadro 1. Secuencias y características fisicoquímicas básicas de los fragmentos del FVIIla3 humano silvestre y los mutantes específicos. Los residuos modificados por mutagénesis dirigida se destacan con negrita y subrayado. Las secuencias correspondientes a las colas de histidinas añadidas para la purificación de las proteínas recombinantes

se destacan en cursiva. Se incluye el peso molecular promedio $\left(P_{m}\right)$, el punto isoeléctrico teórico $(p l)$ y la absorbancia teórica para una solución de $1 \mathrm{~g} / \mathrm{L}$ de la proteína pura a $280 \mathrm{~nm}$ (Abs 0.1\%). Los datos teóricos fueron calculados utilizando la herramienta SIB ExPASy Bioformatics Resources Portal (http://web.expasy.org/protparam/).

\begin{tabular}{|c|c|c|c|c|}
\hline Fragmento & Secuencia de aminoácidos & $\mathrm{P}_{\mathrm{m}}(\mathrm{Da})$ & $\mathrm{pl}$ & $\begin{array}{c}\mathrm{Abs}_{280} \\
0.1 \%\end{array}$ \\
\hline FVIIIa3 & $\begin{array}{c}\text { ASHHHHHHGEITRTTLSDQEEIDYDDTISV } \\
\text { EMKKEDFDIYDEDENQSPRGSGC }\end{array}$ & 6284.55 & 4.54 & 0.474 \\
\hline $\begin{array}{c}\text { FVIIla3 } \\
(\text { R1689Q, G1690P) }\end{array}$ & $\begin{array}{c}\text { ASHHHHHHGEITRTTLQSDQEEIDYDDTISV } \\
\text { EMKKEDFDIYDEDENQSPQPSGC }\end{array}$ & 6296.56 & 4.42 & 0.473 \\
\hline FVIIla3 & ASHHHHHHGEITRTTLQSDQEEIDYDDTISV \\
$($ R1689G, G1690P) & EMKKEDFDIYDEDENQSPGPSGC & 6225.48 & 4.42 & 0.479 \\
\hline
\end{tabular}

Clonaje y sobreexpresión heteróloga de los doble-mutantes del FVIIla3: Se transformaron por triplicado cepas competentes de E. coli One Shot ${ }^{\mathrm{TM}}$ BL21 Star ${ }^{\mathrm{TM}}$ (DE3) pLysS (Invitrogen ${ }^{\mathrm{TM}}$ ) con los plásmidos de expresión que codifican para los fragmentos mutantes del FVIIla3 confirmados por secuenciación, FVIIla3 (R1689Q, G1690P) y FVIIIa3 (R1689G, G1690P), mediante choque térmico. Las cepas transformadas se incubaron en placas de Petri con agar LB, y los antibióticos correspondientes [ampicilina $(100 \mu \mathrm{g} / \mathrm{mL})$ y/o cloranfenicol $(34 \mu \mathrm{g} / \mathrm{mL})$ ], y se incubaron durante toda la noche a $37^{\circ} \mathrm{C}$. Posteriormente, se tomó una colonia bacteriana transformada y se inoculó en $40 \mathrm{~mL}$ de medio LB con los antibióticos correspondientes. El cultivo se incubó durante la noche a $37^{\circ} \mathrm{C}$ con agitación constante de $220 \mathrm{rpm}$. Al día siguiente, se inoculó el precultivo en $400 \mathrm{~mL}$ de medio LB con antibióticos, en un matraz de 2 litros, a $37^{\circ} \mathrm{C}$ con agitación constante de 220 rpm. Una vez alcanzada una densidad óptica (OD) de entre 0.4-0.6 a $600 \mathrm{~nm}$, se indujo la expresión de la proteína recombinante adicionando IPTG a una concentración final de 0.55 $\mathrm{mM}$. El cultivo se incubó durante 6 horas a $37^{\circ} \mathrm{C}$ con agitación constante a $220 \mathrm{rpm}$. Finalmente, se centrifugó el cultivo a 4.000 rpm durante 20 minutos, y el sedimento obtenido se congeló a $-20^{\circ} \mathrm{C}$ hasta su procesamiento.

Extracción y purificación de los doble-mutantes del FVIIla3: La extracción de las proteínas sobreexpresadas en E. coli One Shot ${ }^{\mathrm{TM}} \mathrm{BL21} \mathrm{Star}^{\mathrm{TM}}$ (DE3) pLysS, se realizó mediante lisis celular, resuspendiendo el sedimento bacteriano en $1 \mathrm{~mL}$ de tampón de lisis (40 mM Tris, $\mathrm{pH} \mathrm{8.0,}$ $100 \mathrm{mM} \mathrm{NaCl}, 1 \mathrm{mM}$ PMSF, $1 \mathrm{mM} \beta$-mercaptoetanol, $0.5 \mu \mathrm{g} / \mathrm{mL}$ ADNasa y $20 \mathrm{mM} \mathrm{MgCl}$ ). Se incubó durante 30 minutos en rotor y luego se centrifugó a 12.000 rpm durante 30 minutos a 
$4^{\circ} \mathrm{C}$ para eliminar el material insoluble. A partir de la fracción soluble se purificó la proteína de interés mediante incubación con la resina de afinidad Ni-NTA acoplada a agarosa (Qiagen) a $4^{\circ} \mathrm{C}$ durante 3 horas con agitación suave y constante en un agitador orbital. La suspensión con resina se trasvasó a una columna, se recuperó la fracción no unida a la resina, y se realizaron dos lavados con 10 y 20 mM imidazol, y con $50 \mathrm{mM} \mathrm{NaH}_{2} \mathrm{PO}_{4}, 300 \mathrm{mM} \mathrm{NaCl}$, pH 8.0. Finalmente, se eluyó el material unido de forma específica a la resina empleando $50 \mathrm{mM} \mathrm{NaH}_{2} \mathrm{PO}_{4}, 300 \mathrm{mM}$ $\mathrm{NaCl}, \mathrm{pH}$ 8.0, 500 mM imidazol. La pureza de las muestras purificadas se evaluó mediante SDS-PAGE en geles de poliacrilamida al 12\%. La autenticidad y pureza de los fragmentos recombinantes purificados se comprobó mediante espectrometría de masas con un equipo LTQ-FT Ultra (Thermo Scientific) empleando un nanoelectrospray con modo de polaridad positiva. Los resultados de espectrometría se analizaron con el programa Protein Pilot.

Ensayos de interacción (band shift) de los doble-mutantes del FVIII: Previo a los ensayos de interacción (band shift) y de cristalización los fragmentos FVIIla3 (R1689Q, G1690P) y FVIIla3 (R1689G, G1690P) purificados, estos se dializaron con la finalidad de eliminar el imidazol añadido durante el proceso de purificación, y cambiar el tampón a 10 mM HEPES pH 7.0, 25 $\mathrm{mM} \mathrm{NaCl}$, empleando centrifugación en concentradores (Amicon Ultra 3K). Los ensayos de interacción o desplazamiento de banda ("band shift") se realizaron mediante electroforesis en geles nativos de poliacrilamida al $7.5 \%$. Para el ensayo se incubó una cantidad fija de trombina humana $(15 \mu \mathrm{g})$ con cantidades variables de los fragmentos doble-mutantes del FVIIIa3 en relaciones molares de 1:0.5, 1:1, 1:2, 1:4 y 1:8. Los complejos formados fueron separados de los componentes mediante electroforesis nativa en geles de poliacrilamida al $7.5 \%$, y las bandas de proteína fueron visualizadas con azul de Coomassie.

Ensayos de cristalización de los complejos de la trombina con los doble-mutantes del FVIIla3: Para los ensayos de cristalización, los complejos entre la trombina y las variantes del FVIIIa3 se prepararon con un exceso molar del fragmento recombinante (10:1) en el mismo tampón, a temperatura ambiente por 30 minutos, y se concentraron a $5 \mathrm{mg} / \mathrm{mL}$ utilizando dispositivos Amicon Ultra-15 con poro nominal de $3 \mathrm{kDa}$ (Millipore). Los ensayos de cristalización se llevaron a cabo mediante la técnica de difusión de vapor a $20^{\circ} \mathrm{C}$. Se evaluaron 820 condiciones diferentes de cristalización, incluyendo las 192 condiciones de los kits PAC PROPLEX y PAC PEGRx en placas de 96 pozos (MRC 2-well), empleando un robot Cartesian Microsys, en el Parc Cientific de Barcelona. Se mezcló en cada condición $0.1 \mu \mathrm{L}$ de la disolución del complejo FVIIla3-trombina con $0.1 \mu \mathrm{L}$ de la solución de cristalización, y se dejó equilibrar a través de la fase de vapor con un reservorio de la solución de cristalización. En el CIB, se evaluaron 628 condiciones de cristalización de los kits Morpheus ${ }^{\mathrm{TM}}$ I y II, JCSG-plus ${ }^{\mathrm{TM}}$, ProPlex ${ }^{\mathrm{TM}}$, Structure Screen I y II, Structure Screen 3D y PACTpremier ${ }^{\text {TM }}$ (Molecular Dimensions), en placas de 24 pozos (ComboPlates ${ }^{\mathrm{TM}}$ ). En cada condición se mezcló $1 \mu \mathrm{L}$ de la disolución del complejo respectivo con $1 \mu \mathrm{L}$ de la solución de cristalización, y se equilibró contra $0.5 \mathrm{~mL}$ de la solución de cristalización. Las gotas fueron periódicamente inspeccionadas en busca de cristales con un estereoscopio Nikon SMZ-800N.

\section{Resultados}

Generación de los doble-mutantes del FVIIla3 por mutagénesis dirigida y confirmación por secuenciación de ADN.

A partir del análisis in silico, se identificaron los nucleótidos a ser mutados y se diseñaron los imprimadores para realizar la mutagénesis dirigida y obtener así las secuencias codificantes para las variantes FVIIla3 (R1689Q, G1690P) y FVIIla3 (R1689G, G1690P) (cuadro 2). 
Cuadro 2. Secuencias de imprimadores específicos para la mutagénesis dirigida. Los nucleótidos modificados para inducir los cambios de interés se presentan en mayúscula.

\begin{tabular}{|c|c|c|}
\hline Fragmento modificado & Imprimador & Secuencia \\
\hline \multirow{2}{*}{ FVIIla3 (R1689Q, G1690P) } & Sentido & 5'-accagtctccgcagCCatccggctgctaac-3' \\
\cline { 2 - 3 } & Antisentido & $5^{\prime}$ 'tggtcagaggcgtcGGtaggccgacgattg-3' \\
\hline \multirow{2}{*}{ FVIIla3 (R1689G, G1690P) } & Sentido & $5^{\prime}$ '-ccagtctccgGGgccatccggc-3' \\
\cline { 2 - 3 } & Antisentido & $5^{\prime}$ 'ttttcatcctcatcgtagatgtc-3' \\
\hline
\end{tabular}

Tras realizar el procedimiento de mutagénesis dirigida siguiendo el protocolo establecido por el fabricante del kit se transformaron células con los productos de las reacciones de mutagénesis. Se seleccionaron clones bacterianos y su ADN plasmídico se purificó y secuenció, lo cual permitió confirmar las secuencias de nucleótidos codificantes para los fragmentos FVIIla3 (R1689Q, G1690P) [figura 1] y FVIIla3 (R1689G, G1690P) [datos no mostrados].

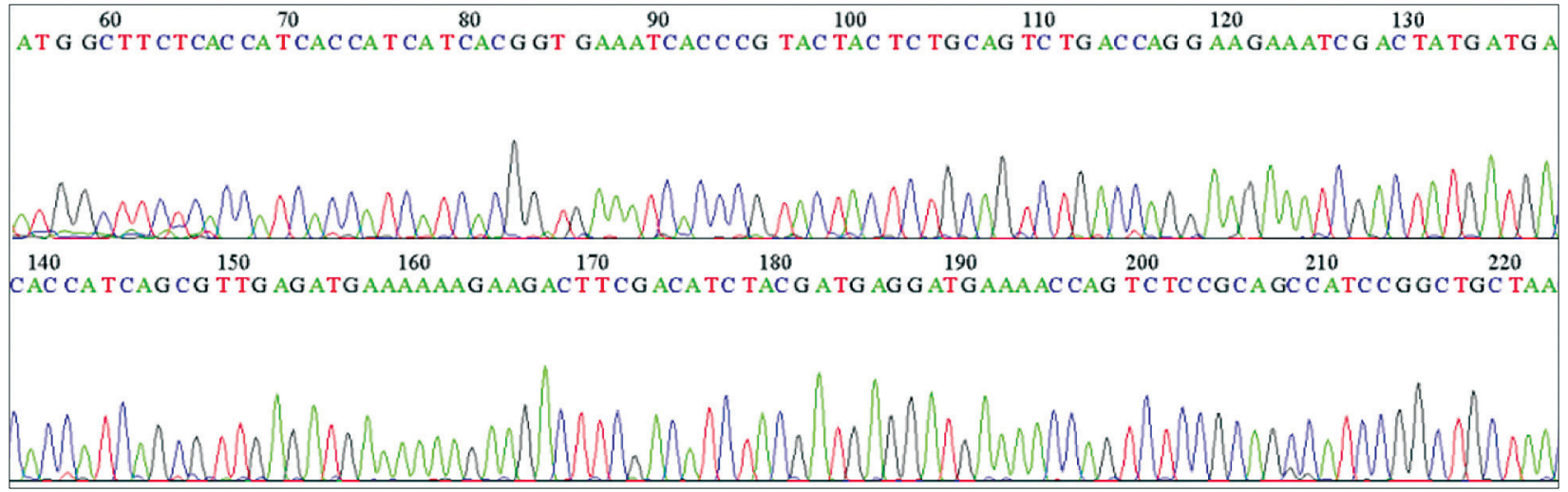

Figura 1. Electroferograma obtenido a partir de la secuenciación del ADN plasmídico que codifica para el fragmento FVIIla3 (R1689Q, G1690P). Los tripletes CAG (nucleótidos 206-208) y CCA (nucleótidos 209-211) corresponden a los residuos de aminoácidos de glutamina $(Q)$ y prolina $(P)$ introducidos en las posiciones P1 y P1' del FVIIla3, respectivamente.

La traducción in silico de las secuencias nucleotídicas obtenidas permitió verificar las secuencias de aminoácidos que se obtendrían al sobreexpresar las variantes FVIIIa3 (R1689Q, G1690P) [figura 2] y FVIIIa3 (R1689G, G1690P) [datos no mostrados].

Los fragmentos FVIIla3 (R1689Q, G1690P) y FVIIla3 (R1689G, G1690P) lograron ser sobreexpresados y purificados a homogeneidad y con un buen rendimiento

Posterior a la extracción y purificación de los fragmentos FVIIla3 (R1689Q, G1690P) [figura 3A] y FVIIIa3 (R1689G, G1690P) [figura 3B] por cromatografía de afinidad (ver "Materiales y Métodos"), los análisis por electroforesis en geles de poliacrilamida desnaturalizantes (SDS-PAGE) permitieron confirmar un alto grado de pureza y un buen rendimiento de la sobreexpresión. 


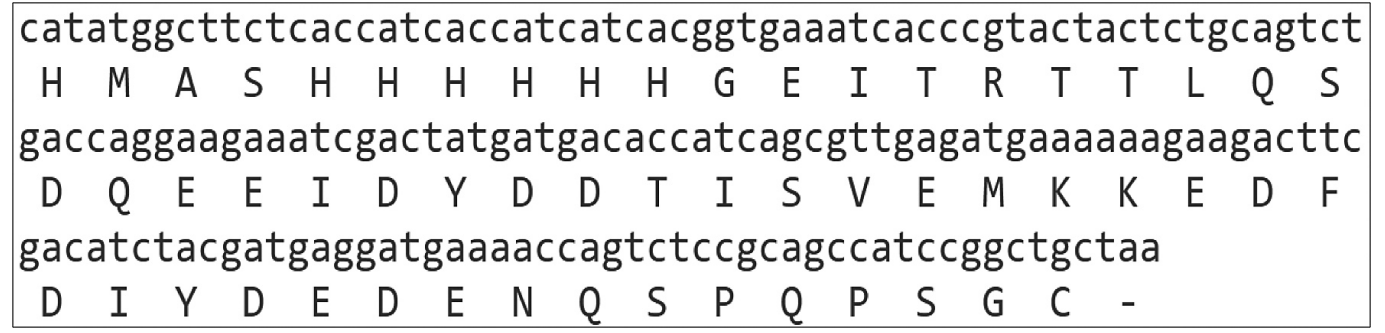

Figura 2. Traducción in silico de la secuencia nucleotídica del FVIIla3 (R1689Q, G1690P). La secuencia aminoacídica correspondiente al fragmento recombinante del doble-mutante FVIIla3 (R1689Q, G1690P) se confirmó con la herramienta SIB ExPASy Bioinformatics Resources Portal. Los nucleótidos se representan con letras minúsculas y los aminoácidos se representan con código de una letra en mayúscula.

A.

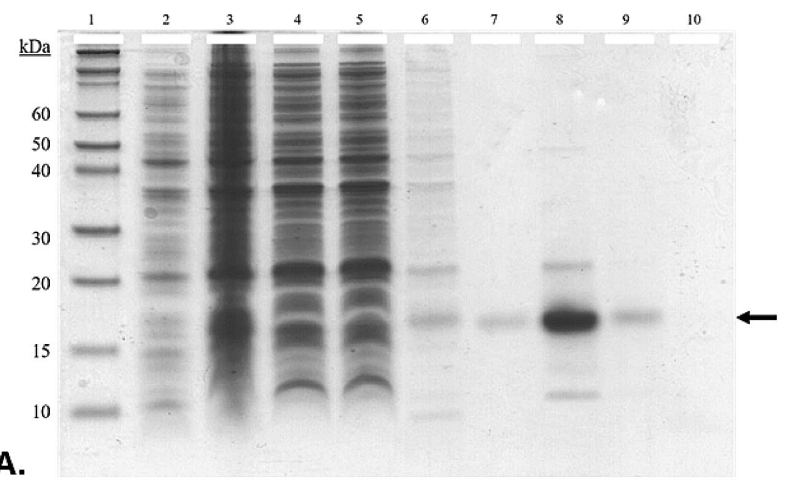

B.

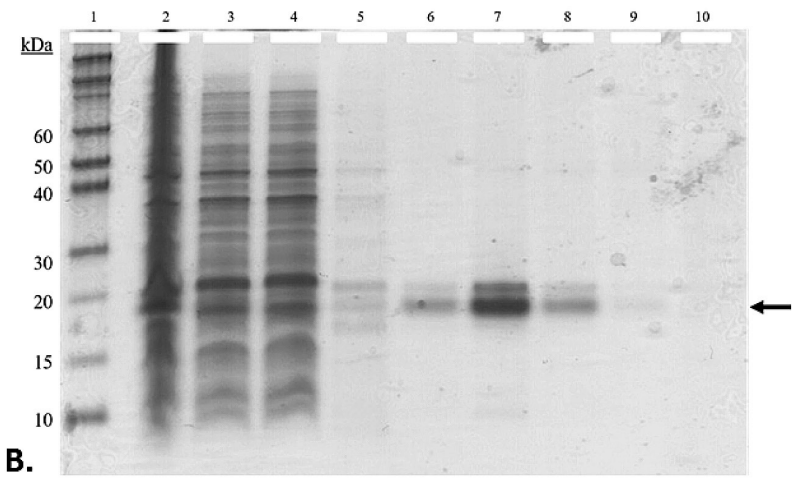

Figura 3. Análisis por SDS-PAGE de la sobreexpresión y purificación de los doble-mutantes FVIIla3 (R1689Q, G1690P) [A] y FVIIla3 (R1689G, G1690P) [B] expresados en E. coli One Shot ${ }^{\text {Tm }}$ BL21 Star ${ }^{\text {Tm }}$ (DE3) pLysS. En el carril 1 se muestra el marcador de peso molecular (Novex Sharp); en los carriles del 2 al 6, se muestran alícuotas de células totales, los extracto solubles en tampón de lisis, las fracciones de proteínas no unidas a la resina de afinidad Ni-NTA, y los lavados sucesivos con $10 \mathrm{mM}$ y $20 \mathrm{mM}$ de imidazol, respectivamente; y en los carriles del 7 al 10 se muestran las eluciones sucesivas con tampón de elución $\left(50 \mathrm{mM} \mathrm{NaH}_{2} \mathrm{PO}_{4}, 300 \mathrm{mM} \mathrm{NaCl}, \mathrm{pH}\right.$ 8.0, $500 \mathrm{mM}$ imidazol). A la izquierda de la imagen se indica el peso correspondiente, en kilodaltons, de las bandas relevantes

del marcador Novex Sharp. A la derecha de la imagen se señala con una flecha la banda de interés.

En los diferentes experimentos de expresión realizados, tras los procesos de purificación y diálisis, se obtuvo un rendimiento promedio de $\sim 1.5 \mathrm{mg}$ de cada fragmento recombinante de interés por litro de cultivo bacteriano. Finalmente, la identidad de las variantes de los fragmentos recombinantes del FVIIla3 purificados se evaluaron mediante espectrometría de masas. Con respecto al FVIIla3 (R1689Q, G1690P), la especie monoisotópica experimental con relación masa/carga (m/z) 6292.7222 Da detectada corresponde exactamente a la secuencia del fragmento recombinante (masa molecular teórica: 6292.72 Da) (figura 4).

La interacción de los doble-mutantes del FVIIla3 con la trombina permitió cristalizar los complejos trombina•FVIIla3

El objetivo de los ensayos de cristalización es obtener cristales de los complejos de la trombina humana con cada uno de los fragmentos recombinantes de interés. Para ello se evaluó la formación de los complejos FVIIla3 (R1689Q, G1690P) y FVIIla3 (R1689G, G1690P) con la trombina, mediante geles de poliacrilamida nativos al 7.5\%. En las figuras 5.A y 5.B se muestra el patrón de bandas resultante, donde se evidencia el desplazamiento de la banda por la 
formación de los complejos de FVIIla3 (R1689Q, G1690P) o FVIIla3 (R1689G, G1690P) con la trombina humana. Nótese la aparición de una nueva banda que parece corresponder al complejo trombina $\bullet F V I I l a 3$ respectivo, debido a la interacción entre las proteínas.

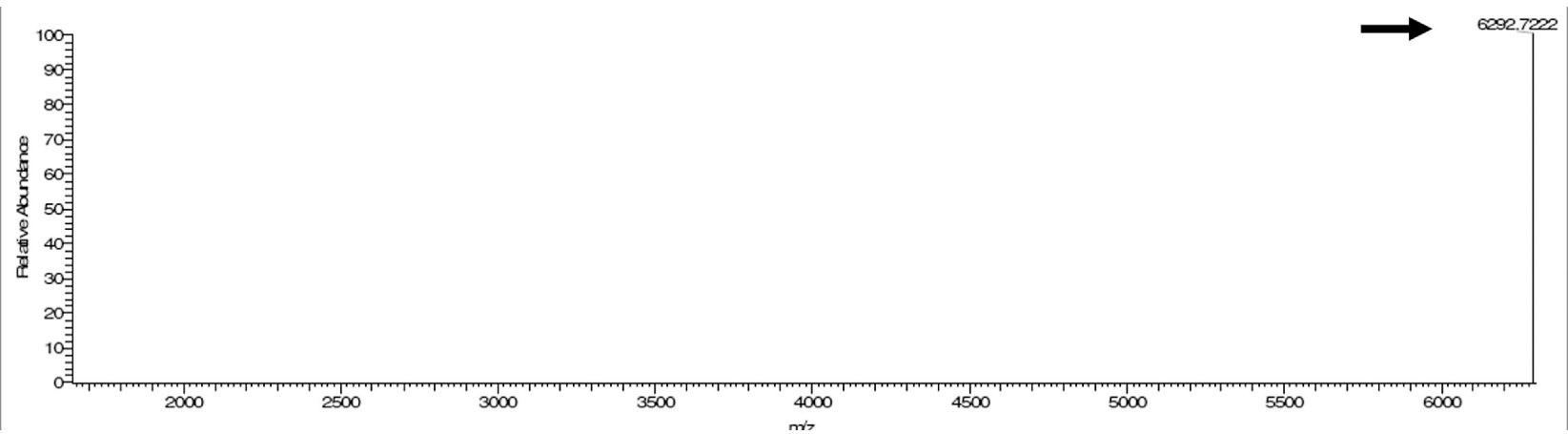

Figura 4. Verificación de la identidad del fragmento recombinante FVIIla3 (R1689Q, G1690P) mediante espectrometría de masas. Especie monoisotópica experimental detectada en el extremo de la ventana espectral empleada (se señala con una flecha). Los experimentos se realizaron con un equipo LTQ-FT Ultra (Thermo Scientific) empleando un nanoelectrospray.

A.

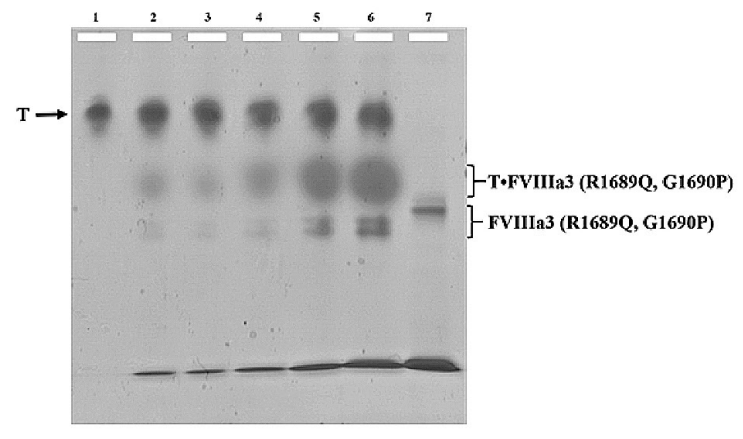

B.

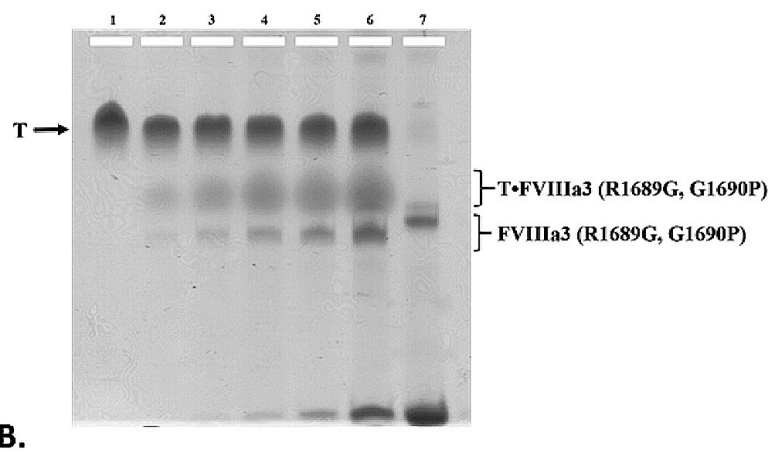

Figura 5. Demostración de la formación de complejos entre el FVIIla3 recombinante y la trombina humana mediante experimentos de band-shift. A. Complejo trombina•FVIIIa3 (R1689Q, G1690P). B. Complejo trombina•FVIIla3 (R1689G, G1690P). Se emplearon geles al 7.5\% de poliacrilamida nativos preparados con tampón Tris-Glicina. En el carril 1 se cargaron $15 \mu \mathrm{g}$ de trombina (T); en los carriles del 2-6 se aplicaron diferentes relaciones molares del fragmento recombinante respecto a la trombina (0.5:1, 1:1, 2:1, 4:1, 8:1, respectivamente); en el carril 7 se cargó una alícuota del doble-mutante de FVIIla3 correspondiente. Nótese en cada caso la aparición de una nueva banda que migra entre las de trombina y la variante del conector ácido del FVIII analizado, y que corresponde al complejo T•FVIIIa3.

Una vez confirmada la formación de los complejos mediante ensayos de band-shift, se prepararon grandes cantidades del complejo de FVIIla3 (R1689Q, G1690P) con la trombina, y se realizó una búsqueda de las condiciones de cristalización utilizando los kits PROPLEX y PEGRx (192 condiciones). A los 3 meses de realizado el screening se observaron posibles microcristales en 7 condiciones del kit PROPLEX, y en 5 condiciones con el kit PEGRx (datos no mostrados). Posteriormente, se prepararon complejos de la trombina tanto con la variante FVIIla3 (R1689Q, G1690P) como con el FVIIla3 (R1689G, G1690P), y se realizó una nueva búsqueda de condiciones de cristalización utilizando los kits de cristalización Morpheus ${ }^{\mathrm{TM}}$, Morpheus $^{\text {TM II, JCSG-plus }}{ }^{T M}$, ProPlex ${ }^{T M}$, Structure Screen I, Structure Screen II, Structure Screen 
3D y PACTpremier ${ }^{\text {Tn }}$. De un total de 628 condiciones evaluadas para cada complejo, en 10 de ellas se obtuvieron mono-cristales de tamaño apropiado. Cabe resaltar que en la condición l-16 de Morpheus ${ }^{\mathrm{TM}}$, compuesta por $0.09 \mathrm{M}$ halógenos, $0.1 \mathrm{M}$ tampón No. 1, pH 6.5, 50\% (v/v) mezcla de precipitantes No. 4, se obtuvieron cristales de ambos complejos. En la figura 6 se muestran algunos ejemplos de los microcristales o precipitados cristalinos que se obtuvieron a partir del screening realizado.
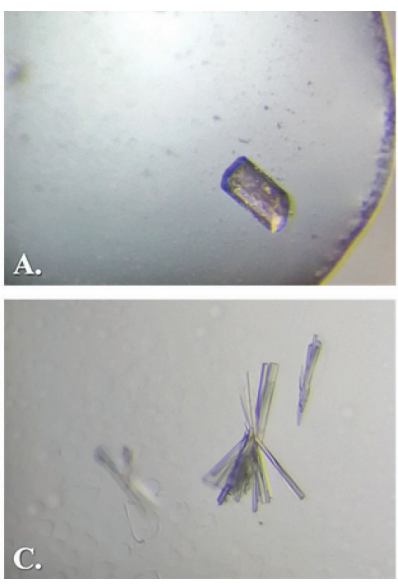
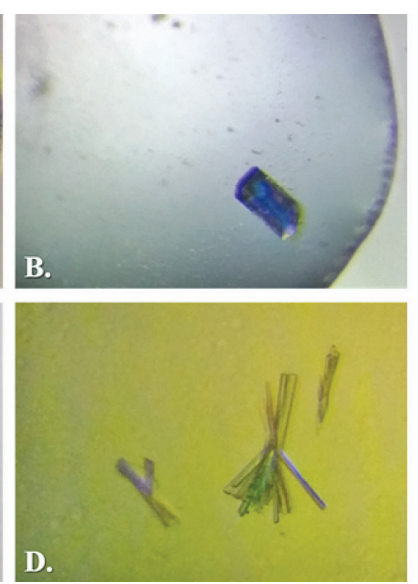
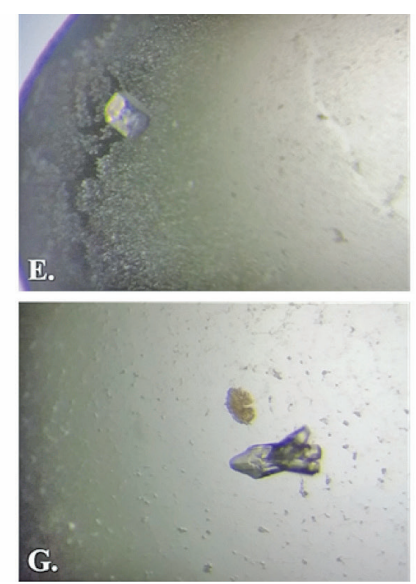
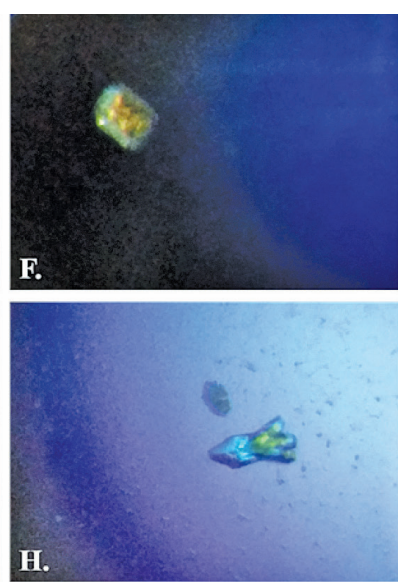

Figura 6. Formaciones cristalinas obtenidas a partir de los complejos de la trombina con los doble-mutantes del FVIIla3. (A-D) Cristales obtenidos a partir del complejo FVIIla3 (R1689Q, G1690P)•trombina. Las imágenes mostradas en los paneles A/C y B/D corresponden a las condiciones I-16 e I-36 del kit Morpheus, iluminadas con luz natural y con luz polarizada, respectivamente. (E-H) Cristales obtenidos a partir del complejo FVIIla3 (R1689G, G1690P)•trombina en las condiciones I-16 (E/F) e I-28 (G/H) de Morpheus, respectivamente. Los paneles F y H muestran las imágenes obtenidas con luz polarizada.

\section{Discusión}

Análisis de la sobreexpresión y purificación de los doble-mutantes del FVIIla3

El requisito imprescindible para poder resolver la estructura 3D de una proteína o complejo proteico utilizando cristalografía de proteínas es la obtención de al menos un cristal de buena calidad para colectar datos de difracción de rayos X. La condición o condiciones en las cuales crecen monocristales no pueden ser predichas, sino que han de ser determinadas a partir de experimentos en los que se explora un gran número de variables $(\mathrm{pH}$, agentes cristalizantes, etc.). Es por ello que el limitante en este tipo de estudios es la necesidad de contar con gran cantidad de proteína, con un alto grado de pureza y homogeneidad [6]. Estudios previos de nuestro grupo [7], evidenciaron la posibilidad de sobreexpresar el conector ácido FVIIla3 como proteína recombinante, en cantidades suficientes para ensayos bioquímicos y de cristalización. Siguiendo las mismas condiciones de expresión empleadas por [7], en este trabajo se lograron sobreexpresar satisfactoriamente los fragmentos recombinantes de dos doble-mutantes del FVIIla3, diseñados para estabilizar su complejo con la proteasa activadora (figuras 3A y 3B). Al analizar mediante SDS-PAGE estas variantes del FVIIla3, con masas moleculares teóricas inferiores a $6.3 \mathrm{kDa}$, observamos que los fragmentos migran como las bandas de 15 y $20 \mathrm{kDa}$ del marcador de peso molecular. Esta migración anómala se debe al fenómeno conocido como "gel shifting", el cual consiste en la notable disminución de la distancia migrada por ciertas proteínas en geles de poliacrilamida-SDS, y se atribuye a las características bioquímicas de las proteínas analizadas y a la composición de los tampones empleados durante los experimentos. 
Estudios previos han demostrado que polipéptidos con un alto contenido de residuos ácidos, como lo es el FVIIla3, repelen las moléculas de SDS, y como consecuencia migran como especies con una masa muy superior a la teórica en geles de poliacrilamida-SDS [8, 9]. Ante esta anomalía, y con el objetivo de confirmar la identidad de las variantes del conector FVIIla3 expresadas, se analizaron las muestras por espectrometría de masas, confirmándose la identidad del fragmento FVIIla3 (R1689Q, G1690P) (figura 4). (En el caso del fragmento FVIIla3 (R1689G, G1690P) se deberá repetir el ensayo, ya que el análisis que se realizó no detectó la especie monoisotópica experimental, probablemente por una ionización deficiente de la muestra). Cabe destacar que el rendimiento promedio obtenido tanto para las variantes FVIIla3 (R1689Q, G1690P) y FVIIla3 (R1689G, G1690P) fue de 1.5 mg por litro de cultivo bacteriano, el cual es tres veces mayor comparado con el rendimiento reportado para la sobreexpresión del conector FVIIla3 silvestre [7].

Efecto de las mutaciones introducidas en la cristalización del complejo trombina•FVIIla3

La resolución de estructuras por medio de la cristalografía de macromoléculas permite la descripción detallada de complejos a nivel molecular e inclusive atómico [10], siendo una de las técnicas básicas para entender el mecanismo de interacción de enzimas en general, y de proteinasas en particular. Por esta razón, la cristalografía fue la técnica escogida para estudiar el mecanismo gracias al cual la trombina proteoliza con gran eficiencia y especificidad los enlaces peptídicos Arg-Xxx en los extremos C-terminales de los conectores interdominio del FVIII (a1, a2 y a3). Para formar un cristal de un complejo proteico, es necesario que este se encuentre de forma estable [11]. Dado que la trombina tiene actividad proteolítica sobre los fragmentos interdominio del FVIII, después de formarse el complejo del FVIIla3 con la proteasa, esta proteoliza el enlace entre el residuo de arginina en la posición 1689 (Arg1689) y la Ser1690, liberándose a continuación el fragmento interdominio y deshaciendo el complejo [4]. Con el objetivo de reducir la capacidad de la trombina para hidrolizar dicho enlace peptídico, se cambió la secuencia del sitio de corte mediante mutagénesis dirigida, tanto de la Arg1689 como de la Ser1690. (En el caso del FVIIIa3 clonado en el vector de expresión, la Arg1689 es seguida de una glicina). Estos dos sitios modificados en el FVIIla3 corresponden, bioquímicamente hablando, a las posiciones $\mathrm{P} 1$ y $\mathrm{P} 1$ ' reconocidos por la trombina en sus sustratos, y al modificarlos se altera la eficiencia del corte mediado por esta proteasa. Los residuos de arginina y serina se han reportado como los más eficientemente reconocidos por la trombina en las posiciones P1 y P1', respectivamente, tal y como se indica en la base de datos de peptidasas MEROPS (https:// www.ebi.ac.uk/merops/). La arginina se caracteriza por tener una cadena lateral terminada en un grupo guanidinio cargado positivamente, que es esencial para su reconocimiento por el residuo Asp ${ }^{189}$ en el fondo del bolsillo S1 de la proteasa. En el FVIIla3 (R1689Q, G1690P), la arginina (R) en la posición P1 fue sustituida por glutamina $(\mathrm{Q})$, un aminoácido polar que tiene una cadena lateral más corta y que termina con un grupo amida no cargado, mientras que para el FVIIla3 (R1689G, G1690P) el cambio es más radical, ya que la glicina (G) es un aminoácido que carece de cadena lateral (sólo un hidrógeno). Por otra parte, en los dos dobles-mutantes estudiados, la glicina $(G)$ de la posición P1' se sustituyó por la prolina $(P)$, un aminoácido no polar y cíclico, dada la ausencia de un grupo $\mathrm{NH}$ libre e impedimentos estéricos hacen que los enlaces Xxx-Pro sean atacados muy poco eficientemente, o no atacados en absoluto. Estos cambios en las posiciones P1 y P1' se supone que sólo alteran las interacciones más directas entre el sitio de corte del FVIIla3 y el sitio activo de la trombina. En apoyo a esta hipótesis, estudios bioquímicos han demostrado que la sustitución Arg>His en la posición P1 disminuye sustancialmente la cinética del corte catalizado por la trombina, pero sin afectar la formación del complejo trombina-factor VIII. Esta observación sugiere que otras regiones de la proteasa serínica, como los llamados 'exositios', gobiernan el anclaje del sustrato FVIII, en el sitio activo de la proteasa, en una conformación óptima para su activación [12]. De esta manera, 
se espera que los cambios realizados dificulten la actividad proteolítica de la trombina sobre los fragmentos del FVIIla3, favoreciendo la formación de complejos más estables, y por tanto, incrementando la posibilidad de observar el conector intacto (no proteolizado) en los cristales crecidos. Para evaluar los resultados de la mutagénesis dirigida y confirmar la formación de los complejos proteicos de interés, previo a los ensayos de cristalización, realizamos ensayos de "band shift". Gracias a estos ensayos logramos confirmar cualitativamente la interacción entre las variantes del FVIIla3 y la trombina en condiciones nativas. A la hora de realizar estos ensayos se consideró la diferencia entre los puntos isoeléctricos (pl) de la trombina (7.0-7.6) y de las dos variantes del FVIIla3 (alrededor de 4.5). Esto permite que las distancias migradas por las bandas correspondientes a la trombina y los fragmentos del FVIIla3 aislados no sólo se diferencien entre sí, sino también de los complejos formados, ya que el aumento en masa se ve compensado por la mayor electronegatividad conferida por el FVIIla3 unido (figuras 5A y 5B). Una vez confirmada la formación cualitativa de los complejos de la trombina con las variantes del FVIIIa3, se realizaron búsquedas sistemáticas iniciales de condiciones de cristalización siguiendo dos estrategias: 1) empleando un robot que dispensa gotas con volúmenes de nanolitros ( $\mathrm{nL}$ ) (en la Plataforma de Cristalografía Automatizada del Parc Cientific de Barcelona), y 2) dispensando manualmente microgotas ( $\mu \mathrm{L}$ ) (en el CIB). La estrategia automatizada permitió encontrar 12 condiciones en las cuales se observaron formaciones microcristalinas, mientras que la estrategia de micro-gotas, permitió encontrar formas cristalinas de mayor tamaño en 10 condiciones diferentes (figura 6). Cabe destacar en particular la condición l-16 del kit de Morpheus [0.09 M halógenos, $0.1 \mathrm{M}$ tampón No.1, pH 6.5 y 50\% (v/v) mezcla de precipitantes No.4], en la que se obtuvieron los cristales de mayor tamaño para ambos complejos. A partir de los resultados del screening primario, es posible optimizar parámetros como $\mathrm{pH}$, concentraciones de agentes precipitantes y de proteína, etc. alrededor de las condiciones más prometedoras (screening secundarios), con el objetivo de mejorar la calidad de los cristales obtenidos. Esto a su vez, permitirá colectar mejores conjuntos de datos de difracción de rayos $X$, que permitan resolver y refinar las estructura 3D de las proteínas presentes en el cristal a la mayor resolución posible. En el caso concreto de este proyecto, esto permitiría eventualmente conocer a nivel atómico las regiones del FVIIIa3 que interaccionan con la trombina, tanto con el sitio activo como con los exositios I y II, y con ello, comprender el mecanismo de activación del FVIII mediado por la trombina.

\section{Conclusiones}

Se lograron generar los dobles-mutantes del conector interdominio del FVIIla3 humano, a saber, FVIIla3 (R1689Q, G1690P) y FVIIla3 (R1689G, G1690P) mediante mutagénesis dirigida y se confirmaron mediante secuenciación de ADN. Ambos dobles-mutantes del FVIIla3 se sobreexpresaron de forma heteróloga en E. coli y se purificaron a homogeneidad con alto rendimiento. La formación de los complejos de las variantes mutadas del FVIIla3 con la trombina se confirmaron mediante band shift, y la búsqueda de condiciones de cristalización permitió obtener algún tipo de precipitado cristalino en 22 condiciones, 10 de las cuales lograron ser optimizadas para crecer mono-cristales de calidad para posteriores ensayos de difracción con rayos $X$.

\section{Agradecimientos}

A la Vicerrectoría de Vida Estudiantil por apoyar el financiamiento de la estancia científica de J.R-M. en la Unidad de Bases Moleculares de las Enfermedades del Institut d'Investigació Biomèdica, del Hospital de la Santa Creu i Sant Pau (Barcelona, España), y a la Vicerrectoría de Investigación y Extensión del TEC por el financiamiento del proyecto (CF 1510078). 


\section{Referencias}

[1] A. Timmis et al., "European Society of Cardiology: Cardiovascular Disease Statistics 2017", Brussels: European Heart Journal, vol. 39, no. 7, pp. 508-579, 2018.

[2] "Enfermedades cardiovasculares", Organización Mundial de la Salud, 2014.

[3] B. Furie, B y B. C. Furie, "Mechanisms of thrombus formation", N. Engl. J. Med., vol. 359, no. 9, pp. 938-949, 2008. https://doi.org/10.1056/NEJMra0801082

[4] P. J. Fay, "Activation of factor VIII and mechanisms of cofactor action". Blood Rev., vol. 18, no. 1, pp. 1-15, 2004. https://doi.org/10.1016/S0268-960X(03)00025-0

[5] P. W. Rose et al., "The RCSB Protein Data Bank: views of structural biology for basic and applied research and education", Nucleic Acids Res., vol. 43, no. D1, pp. 345-356, 2014. https://doi.org/10.1093/nar/gku1214

[6] B. Rupp, "Biomolecular crystallography: principles, practice, and application to structural biology", Garland Science, 2009.

[7] E. Hernández-Carvajal et al. "Producción heteróloga y caracterización bioquímica del procoagulante humano Factor VIII para ensayos de cristalización de macromoléculas proteicas”, Tecnol. Marcha, vol. 29, no. 4, pp. 78-91, 2017.

http://dx.doi.org/10.18845/tm.v29i4.3039

[8] A. Rath et al., "Detergent binding explains anomalous SDS-PAGE migration of membrane proteins", Proc. Natl. Acad. Sci. U.S.A., vol. 106, no. 6, pp. 1760-1765, 2009. https://doi.org/10.1073/pnas.0813167106

[9] Y. Shi et al., "Abnormal SDS-PAGE migration of cytosolic proteins can identify domains and mechanisms that control surfactant binding", Protein Sci. vol. 21, no. 8, pp. 1197-1209, 2012. https://doi.org/10.1002/pro.2107

[10] G. C. Lander, H. R. Saibil y E. Nogales. "Go hybrid: EM, crystallography, and beyond", Curr. Opin. Struct. Biol., vol. 22, no. 5, pp. 627-635, 2012. https://doi.org/10.1016/j.sbi.2012.07.006

[11] A. P. Turnbull y P. Emsley, "Studying protein-ligand interactions using X-ray crystallography". Protein-Ligand Interactions: Methods and Applications, pp. 457-477, 2013. https://doi.org/10.1007/978-1-62703-398-5_17

[12] K. Nogami et al., "Thrombin-catalyzed activation of factor VIII with His substituted for Arg372 at the P1 site", Blood, vol. 105, no. 11, pp. 4362-4368, 2005. https://doi.org/10.1182/blood-2004-10-3939 